\title{
Probing dopants in wide semiconductor quantum point contacts
}

Irina I. Yakimenko and Karl-Fredrik Berggren

\author{
Linköping University Post Print
}

\section{Tweet}

N.B.: When citing this work, cite the original article.

Original Publication:

Irina I. Yakimenko and Karl-Fredrik Berggren, Probing dopants in wide semiconductor quantum point contacts, 2016, Journal of Physics, (28), 10, 1-10.

http://dx.doi.org/10.1088/0953-8984/28/10/105801

Copyright: IOP Publishing

http://www.iop.org/

Postprint available at: Linköping University Electronic Press

http://urn.kb.se/resolve?urn=urn:nbn:se:liu:diva-125790 


\title{
Probing dopants in wide semiconductor quantum point contacts
}

\author{
I.I. Yakimenko and K.-F. Berggren \\ Department of Physics, Chemistry and Biology, Linköping University, SE-58183 \\ Linköping, Sweden
}

\begin{abstract}
Effects of randomly distributed impurities on conductance, spin polarization and electron localization in realistic gated semiconductor quantum point contacts (QPCs) have been simulated numerically. To this end density functional theory in local spin-density approximation (LSDA) has been used. In the case when the donor layer is embedded far from the two-dimensional electron gas (2DEG) the electrostatic confinement potential exhibits the conventional parabolic form, and thus the usual ballistic transport phenomena take place both in the devices with split gates alone and with an additional metallic gate on the top. In the opposite case, i.e., when the randomly distributed donors are placed not far away from the 2DEG layer, there are drastic changes like the localization of electrons in the vicinity of confinement potential minima which give rise to fluctuations in conductance and resonances. The conductance as a function of the voltage applied to the top gate for asymmetrically charged split gates has been calculated. In this case resonances in conductance caused by randomly distributed donors are shifted and decrease in amplitude while the anomalies caused by interaction effects remain unmodified. It has been also shown that for a wide QPC the polarization can appear in the form of stripes. The importance of partial ionization of the random donors and the possibility of short range order among the ionized donors are emphasized. The motivation for this work is to critically evaluate the nature of impurities and how to guide the design of high-mobility devices.
\end{abstract}

73.61-r, 71.15Mb, 71.70.Gm

\section{Introduction}

Electron transport in ballistic semiconductor quantum wires (QWs) and QPCs attracts considerable interest because of the existence of intriguing many-body effects which can manifest themselves in the quantized conductance patterns known as the 0.7 conductance anomaly (or feature) and its analogs [1]-[4]. Spin correlations are widely believed to be involved in these anomalies. The idea of spin polarization in QPCs originates from Pepper and co-workers [5, 6]. Later this idea has been developed theoretically by the group of Berggren [7]-[12]. In this model the anomalous conductance plateaux in the vicinity of $0.7 \times 2 e^{2} / h$ is due to some remnant (or spontaneous) spin polarization of the 2DEG in the QPC region. Such a concept has been extensively used for the interpretation of a variety of experimental results for anomalous conductance phenomena in QPCs and QWs [2]. The spin-polarization model is consistent with a recent exact diagonalization study of a model constriction in a few-electron two-dimensional wire [13].

There is also another (dynamic) version of the spin-polarization model which has been argued by Meir et al. [14, 15]. In this case one starts from the similarity between the 0.7 feature in QPCs and Kondo effect in quantum dots [16]. The model is thus essentially based on the assumption that there is a local magnetic moment within a QPC, presumably originating from spin correlations as above. Thus there 
is also a number of related theoretical and experimental studies of bound states at pinch-off, for example in [17]-[20]. Here we focus, however, on the crucial role of disorder among the dopants and its effect on conductance. More recently there is also a model based on one-dimensional van Hove singularities [21] suggesting that there is only a singlet ground state in stricktly one-dimensional QPCs. This model, however, does not apply to the very wide QPCs discussed here and depicted in Figure 1.

Hew et al. [22] have studied the possibility for the formation of Wigner crystals within QPCs with a shallow confinement. In contrast to the case of a strong confinement when the conductance is characterized by the steps of integer $2 e^{2} / h$, these plateaux become suppressed and conductance jumps directly from 0 to $4 e^{2} / h$ when a wide QPC populates. From experiments, it has been suggested that due to Coulomb interactions the system seems to be driven into a double-row configuration state with effectively two channels contributing to the conductance. Moreover, subsequent research [23] shows direct evidence both of the two-row configuration and indications of level rearrangements appearing at different confinement potentials and electron densities conditions in the presence of an applied external magnetic field.

Theoretical studies of a quantum ring and a one-dimensional quantum wire using quantum Monte-Carlo simulation have shown the existence of antiferromagnetic localized states [24] and the formation of a zig-zag chain [25] in a low electron density regime. In our paper [26] we have studied a special cylindrical semiconductor stripe at ultra-low electron densities. We have shown the interaction-induced bifurcation of the system into a spin zig-zag configuration. The results of our modeling within LSDA are in good agreement with the results of Monte-Carlo simulation in [24]. Structures of such kind may be responsible for the anomalous behavior of the quantized conductance of a quantum wire in a shallow confinement limit, as discussed by Hew et al [22]. In a recent paper [27] we have shown that in the case of soft confinement potentials the degree of localization is small and only traces of partial electron localization in the middle and on the edges of a QPC in the pinch-off regime may be observed. On the other hand, strong confinement potential, low electron density in the leads and implant or top gate geometry favor electron localization [27].

It should be noticed, however, that there are some other unavoidable features which may have a strong influence on the conductance described above. Randomly distributed ionized donors is a typical case. According to previous studies [28][31] the presence of disorder among such donors leads to random fluctuation in the electrostatic potential that in turn results in the distortion of the conductance plateaux. For lithographically longer QPCs this effect becomes stronger up to a total smearing of the plateaux. In addition, due to random impurities conductance may be modified by the appearance of new resonances caused by tunneling of electrons between leads and localized electron states within QPCs. This is important as it comes to applications. For example, homogeneity and order of an assembly of nanostructures are crucial for their use in large-scale electronic or optical systems [32]. The following explicit case is intriguing.

Thus, a statistical study of an array of 256 nominally identical QPCs [33] has demonstrated the crucial role of fluctuations of the potential profile for QPC. It was found that only $41 \%$ of total set of QPCs maintains the usual step-like conductance structure with the 0.7 anomaly. In most of the QPCs potential variations were 
caused by effects of disorder in spite of the fact that all QPCs were fabricated under the same conditions on the same wafer (see also [34]). In this respect theoretical modeling of realistic heterostructures may be helpful for qualitative estimation of different mechanisms contributing to conductance.

From the early 1990s it has been widely recognized that impurity scattering may lead, as mentioned, to the vanishing of integer conductance plateaux in symmetrically biased QPCs [35]-[37]. In particular, in the study of the GaAs/AlGaAs QPCs with random impurities situated in a delta-layer $42 \mathrm{~nm}$ above the channel [36] the degradation of conductance plateaux has been observed for $200 \mathrm{~nm}$ long channels which became more pronounced for $600 \mathrm{~nm}$ long QPC channels in the case of increased density of impurities. In [37] one discusses a systematic study of the dependence of conductance fluctuations on the length of QPC as well as fluctuation statistics for a QPC with a parabolic confinement potential. However, all these studies have been restricted to the Thomas-Fermi approximation, and thus the effects of electron correlation and realistic confinement potentials were not within reach. The purpose here is to go beyond this model by using the more accurate LSDA. The validity of this model has been approved in various studies, for example, $[14,15,24,26]$. There are more elaborated methods to treat correlations but in a case like ours one must make a computational compromise. Another important problem that we will address here is the nature of impurity distributions and degree of ionization of donors.

The role of a disorder has shown to be very important also in InAs/InGaAs heterostructures. For example, in the study of an in-plane gated InGaAs QPC with the use of scanning gate microscopy [38]-[39] it was clearly shown that quantum interference patterns are caused by random potential fluctuations. Such structures have recently attracted attention in connection with spintronics applications where a QPC is a main working element (such as spin-polarizers and spin valves). A spinpolarized current is created by means of lateral spin-orbit coupling in an asymmetric gated QPCs [40]-[42]. It has been shown in [43] that the number, location and shape of conductance anomalies below the first conductance plateaux are strongly dependent on the nature and location of impurities. This is of practical importance because spin polarization should be very well reproducible in a large number of switching elements for spintronics circuits as indicated above. At the same time disorder of this type provides ideal models for fundamental studies of wave-chaotic transport [44].

Recently [45] the idea of using lateral spin-orbit coupling has been exploited for the implementation of all-electric Datta-Das spin-FET [46] in GaAs/AlGaAs heterostructure. This device could be potentially used in all-electric spin-based sensors, spin filters, logic elements and building blocks for quantum computing [47]. The high expectation for applications of proposed spin-FET motivates studies of influence of imperfections on its functionality.

Below we present a model of a GaAs/AlGaAs QPC with randomly distributed impurities (Section II) and discuss numerical results concerning the role of randomly distributed fully [35] and partially ionized donors [48], [49] on electron transport in GaAs/AlGaAs heterostructures of different geometry revealed within the framework of the LSDA (Section III). Conclusions are given in Section IV. 


\section{Model of heterostructure with randomly distributed donors}

Two-dimensional electron gas residing below the surface of a gated modulationdoped GaAs/AlGaAs heterostructure can be formed by proper combination of semiconductor materials, patterned gates, doping and applied gate voltages. It can further be shaped by a negative voltage applied to the metallic gates on the top of the structure. In this way quantum constrictions like QWs and QPCs can be implemented as described in [3] and [50]. The electric current characteristics is measured experimentally if a small source-drain bias is applied at the two edges of this structure. To find them theoretically we will start (as in our previous papers [12] and [27]) from the two-dimensional Kohn-Sham equations:

$$
\left[-\frac{\hbar^{2}}{2 m^{*}} \nabla^{2}+V_{e f f}^{\sigma}(\mathbf{r})\right] \varphi_{i}^{\sigma}(\mathbf{r})=E_{i}^{\sigma} \varphi_{i}^{\sigma}(\mathbf{r}),
$$

where $\mathbf{r}=(x, y)$ is a position vector in a two-dimensional space, and $\varphi_{i}^{\sigma}(\mathbf{r})$ is the wave function for the electron of the $\sigma$-spin orientation in the state with the eigenenergy $E_{i}^{\sigma}$. Effective potential in Eq. (1) is a sum of electrostatic confinement $V_{\text {conf }}$, Hartree $V_{H}$, and exchange-correlation $V_{x c}^{\sigma}$ potentials:

$$
V_{e f f}^{\sigma}(\mathbf{r})=V_{\text {conf }}(\mathbf{r})+V_{H}(\mathbf{r})+V_{x c}^{\sigma}(\mathbf{r}) .
$$

Electrostatic confinement potential is given by

$$
V_{\text {conf }}(\mathbf{r})=-e V_{g}(y)-e V_{s g}(\mathbf{r})-e V_{r d}(\mathbf{r})-e V_{s},
$$

where $V_{g}$ is the potential from the two semi-infinite metallic gates as in Fig. 1, held at constant voltage and separated by a slit of the lithographic width of $400 \mathrm{~nm}$ (analytical expression for this potential can be found in [51]); in addition the Fermi energy of the two reservoirs is pinned to surface states at the metal-semiconductor interface. Choosing $V_{g}=-0.7 \mathrm{~V}$ we obtain electron densities in the reservoirs which match experiments. $V_{s g}$ is the potential from the split gates calculated by numerical integration over the surface of split gates (lithographic length and width of split gates are $200 \mathrm{~nm}$ which are typical values for real devices [4]), $V_{r d}(\mathbf{r})$ is the contribution from donors and $V_{s}$ is the potential from surface states. To study the effect of disorder we will follow the model proposed in the paper [52]. We thus assume that ionized donors are distributed over a slab (in Fig. 1) and define the potential $V_{r d}(\mathbf{r})$ in the form

$$
V_{r d}(\mathbf{r})=V_{d}(\mathbf{r})+\delta V_{d}(\mathbf{r})
$$

where $V_{d}(\mathbf{r})$ is the conventional potential from an infinite slab with a given uniform impurity donor density $n_{i}$. The term $\delta V_{d}(\mathbf{r})$ is the deviation of the impurity random donor potential, $V_{i r d}(\mathbf{r})$, created by a slab of finite size for a particular case of the uniform distribution of donors, $V_{i r d}^{0}(\mathbf{r})$, that is,

$$
\delta V_{d}(\mathbf{r})=V_{i r d}(\mathbf{r})-V_{i r d}^{0}(\mathbf{r}) .
$$

Both potentials on the r.h.s. of Eq. (5) can be calculated using the general expression

$$
V_{i r d}(\mathbf{r}, z)=\frac{1}{4 \pi \epsilon_{0} \epsilon} \sum_{j}\left(\frac{1}{\left(\left(z-z_{j}\right)^{2}+\left|\mathbf{r}-\mathbf{r}_{j}\right|^{2}\right)^{1 / 2}}-\frac{1}{\left(\left(2 z-z_{j}\right)^{2}+\left|\mathbf{r}-\mathbf{r}_{j}\right|^{2}\right)^{1 / 2}}\right) .
$$


where $\mathbf{r}_{j}=\left(x_{j}, y_{j}\right)$ and $z_{j}$ define a position of the $j$ :th ionized donor within a slab, $z$ is the distance from the top of the structure to 2DEG, and $\epsilon$ is the dielectric constant (equal to 12.9 for GaAs). The second term in this expression counts the contribution from mirror charges. The distance $s$ between 2DEG and donor layer was varied from $10 \mathrm{~nm}$ to $100 \mathrm{~nm}$ in order to estimate the influence of the completely randomly distributed donors on the confinement potential. To calculate the first term in Eq. (4) we put donor density $n_{i}=6 \cdot 10^{17} \mathrm{~cm}^{-3}$ and assume in a first step that all donors are ionized as frequently expected to occur upon illumination. In a second step we will assume that there may be a fraction of neutral donors and partial short range order. The Hartree term $V_{H}$ in Eq. (2) is implied to be calculated numerically with inclusion of contributions from mirror charges. Finally, for the exchange-correlation potential $V_{x c}$ we apply the parameterization scheme from [53].

The spin-dependent set of equations (1) must be solved in an iterative way, a process that needs some care to allow for both non-magnetic and magnetic solutions. Consider the case that there is no external magnetic field $B$. If the iterations are initiated with trial states that are doubly occupied, i.e. the total state is nonmagnetic, also the final converged state will remain non-magnetic. However, to give a way to spin-symmetry breaking and possible magnetic ground state solutions with enhanced magnetization due to electron interactions we must trigger the system in that direction right from the start of the iterations. One straightforward way to do so is to let (some) levels be singly occupied. The total trial state is in this case assumed to be spin-polarized. This does not mean, however, that also the final self-consistent state turns out to be magnetic. That would depend on Fermi energy, type of confinement, etc. A computationally convenient version of this scheme is to introduce a weak Zeeman term $g \mu_{B} B \sigma$ into Eq. (1) as in, for example, [12]. Typically one may choose a Zeeman splitting of $10^{-6} \mathrm{eV}$. The Zeeman term in Eq. (1) may, however, be switched off already after the first iteration or so without affecting the final solution except for the possibility of symmetry breaking. The iteration process would also converge equally well if we let the initial exchange and correlation potential in Eq. (2) be randomized in each computational point corresponding to positive and negative B-fields, or if we choose a non-equilibrium set of occupation numbers of the trial states, still keeping the net polarization equal zero. Thus magnetized solutions are, whenever they occur, very robust.

It is sometimes argued that the introduction of an external magnetic field is an artificial trick without physical motivation. One may recall, however, that in a real device there are local magnetic fluctuations due to wiring, vortical currents associated with impurities etc. One may also recall that the background geomagnetic field is 30-60 $\mu \mathrm{T}$, field at which the Zeeman term above may induce magneteic solutions at zero temperature [54]. When a real sizable magnetic field is applied to the system the concerns above are, of course, irrelevant.

When solving the self-consistent Kohn-Sham equations (1) we impose periodic boundary conditions

$$
\varphi_{k}^{\sigma}\left(x+T_{x}, y\right)=\varphi_{k}^{\sigma}(x, y) \mathrm{e}^{i k T_{x}},
$$

where $T_{x}$ is the period in longitudinal $x$-direction (400 $\mathrm{nm}$ in our calculations), 
$\sigma= \pm 1 / 2$ is the spin orientation, and $k$ is the wave vector,

$$
k=\frac{2 \pi m}{M T_{x}} .
$$

Here $m$ is an integer belonging to the first Brillouin zone, $-M / 2<m \leq M / 2, M$ being the number of unit cells (10 in our calculations); hence $\varphi_{k}^{\sigma}(\mathbf{r})$ is normalized to $1 / M$ over the unit cell.

We have studied a QPC of lithographic length $200 \mathrm{~nm}$ and width of $200 \mathrm{~nm}$. As an input for the iterative process we use the total effective potential calculated in the Thomas-Fermi approximation. At each consecutive iterative step the potential is updated in accordance with the electron density for this step,

$$
\rho^{\sigma}(\mathbf{r})=\sum_{k} n\left(E_{k}^{\sigma}, \mu, T\right)\left|\varphi_{k}^{\sigma}(\mathbf{r})\right|^{2}
$$

where $n(E, \mu, T)=1 /\left(1+\exp \left((E-\mu) / k_{B} T\right)\right.$ is the Fermi-Dirac occupation number for temperature $\mathrm{T}$ and chemical potential $\mu$ which is here set equal to zero. The iterative procedure is terminated when the electron densities become identical within a given tolerance $\left(10^{-5}\right.$ in our calculations).

The spin polarization of the system is calculated as the difference between densities for up- and down-spin electrons at the end of iterative process

$$
p(\mathbf{r})=\rho^{\sigma}(\mathbf{r})-\rho^{-\sigma}(\mathbf{r}) .
$$

Using the self-consistent solutions of the Kohn-Sham equations and the definition of the probability current density for spin $\sigma$,

$$
J^{\sigma}=\frac{\hbar}{2 m i} \sum_{k \geq 0}\left[\varphi_{k}^{\sigma *} \frac{\partial \varphi_{k}^{\sigma}}{\partial x}-\frac{\partial \varphi_{k}^{\sigma *}}{\partial x} \varphi_{k}^{\sigma}\right]\left(n\left(E_{k}^{\sigma}, \mu+e V_{s d} / 2, T\right)-n\left(E_{k}^{\sigma}, \mu-e V_{s d} / 2, T\right)\right)
$$

( $V_{s d}$ is the bias voltage between the source and drain), we were able to calculate the main quantities of our interest, that is, the total current through a given QPC and its conductance. The former can be obtained from Eq. (11) by summation over $\sigma$ and integration over transversal $(y)$ direction, while the differential conduction is simply the ratio between the total current and source-drain voltage at small voltages.

The computations invite naturally to parallelism in a numerical code. We therefore use an algorithm adapted for parallel high performance cluster with distributed memory (for more details see Ref. [27]).

\section{Results of numerical simulations}

To study the influence of random donors on the total electrostatic potential and conductance, we have performed numerical simulations for three distances between the 2DEG and donor layer in Fig.1: $s=10 \mathrm{~nm}, s=50 \mathrm{~nm}$ and $s=100 \mathrm{~nm}$. In all cases the donors are assumed to be randomly distributed in a slab of the height $38 \mathrm{~nm}$, length $400 \mathrm{~nm}$ and width $400 \mathrm{~nm}$. The thickness of the top undoped cap layer is $24 \mathrm{~nm}$. The distance between the metal surface and 2DEG $z$ is thus varied as 72,112 and $162 \mathrm{~nm}$ for the three cases which implies that confinement potential 
$V_{\text {conf }}(\mathbf{r})$ becomes more shallow the deeper 2DEG layer is embedded. Note that these values for $z$ are typical for realistic QPCs $[2,31]$.

The main purpose of these calculations is to specify practical design parameters for which the influence of random donors may be neglected or minimized. We are also interested in specific effects that may be caused by the type of donor randomness, for example, occurrence of partial ionization and short range order [48]-[49].

\section{A) The case of $s=10 \mathrm{~nm}$ for fully randomized ionized impurities:}

We start simulations with the case of symmetrically charged split gates. The results of the calculations of confinement, total potentials and electron densities for this case are shown in Figs. 2 and 3. Fig. 2a shows a distinct deviation from the conventional parabolic form for a confinement potential in the case when $V_{s g}=-1.6 \mathrm{eV}$. This is seen as additional barriers and dents along the channel. The corresponding total potential for the same gate voltage shown in Fig. $2 \mathrm{~b}$ is more flat but a transverse barrier appears at the center of the QPC. There are also local minima in a total potential profile that allow electron localization within these regions. At higher $V_{s g}=-1 \mathrm{eV}$ the potential profile becomes even more randomized (Fig. 2c).

In Fig. 3 we plot the total electron densities at different split gates voltages $V_{s g}$. At $V_{s g}=-1.6 \mathrm{eV}$ (Fig. 3a) the pinch-off regime is realized. Conductance for this case is $G=0.005 G_{0}\left(G_{0}=2 e^{2} / h\right)$. In this regime localized states reside near the two openings of a QPC (as argued in [12] and [15]). For $V_{s g}=-1.4 \mathrm{eV}$ (Fig. 3b) the conductance is approximately $G=0.2 G_{0}$ and one observes pronounced peaks in electron density along a QPC. At $V_{s g}=-1 \mathrm{eV}$ (Fig. 3c) the conductance is $G \sim 0.75 G_{0}$ and the corresponding peaks indicate the formation of localized states along the QPC. The impact of the random potential is evidently strong in this case.

The total conductance (curve (a) in Fig.4) has the characteristic resonance peaks near $G=0.2 G_{0}$ and $G=0.75 G_{0}$ that may be traced to tunneling of electrons through localized states inside of the QPC. Curves (b) and (c) in the same figure correspond to the partial conductance for up- and down-spin electrons. We see that above $G=0.75 G_{0}$ there is no usual integer conductance plateaux which agrees with the fact that for split gates potential $V_{s g}>-0.6 \mathrm{eV}$ the confinement is essentially weaker and randomized. In the vicinity of $G=0.75 G_{0}$ there is a peak which differs from the conventional 0.7 anomaly and may be also treated as an effect pertaining to phenomena caused by a disorder. In general, we can conclude that in the case when distance between 2DEG and donor layer is $s=10 \mathrm{~nm}$ resonances on a conductance should be associated rather with the presence of localized states than with electrons correlation effects.

In the next set of simulations for $\mathrm{s}=10 \mathrm{~nm}$ we place a top metallic gate above the split-gates and apply a top gate voltage $V_{t g}$ that corresponds to the configuration of type which has been implemented in [31] to study the effects of disorder and interactions in QWs. Conductance curves as a function of top gate voltages $V_{t g}$ are shown in Fig. 5 for the case of the symmetrically set split-gate voltages at a) $\left.V_{s g}=-0.8 \mathrm{eV}, \mathrm{b}\right) V_{s g}=-1 \mathrm{eV}$, and c) $V_{s g}=-1.2 \mathrm{eV}$. Fig. 5 gives clear evidence for a new resonance near $G=0.6 G_{0}$ (the resonance position is shifted with changing the value of $V_{s g}$ ).

The influence of an asymmetry in the right, $V_{s g 1}$, and left, $V_{s g 2}$, split-gate volt- 
ages is illustrated in Fig. 6. As a reference, one can use Fig. 6a which is drawn for the symmetrically charged split-gates, while Fig. $6 \mathrm{~b}$ and $6 \mathrm{c}$ correspond to the asymmetric cases: (b) $V_{s g 1}=-1.0, V_{s g 2}=-1.2 \mathrm{eV}$ and (c) $V_{s g 1}=-1.2 \mathrm{eV}, V_{s g 2}=-1.0$ $\mathrm{eV}$, so that the off-set $\Delta V=V_{s g 1}-V_{s g 2}$ equals $0.2 \mathrm{eV}$ in Fig. $6 \mathrm{~b}$ and $-0.2 \mathrm{eV}$ in Fig. 6c. As it is seen, the resonant peaks are shifted and (or) diminished in amplitude depending on the sign of the off-set $\Delta V$ that should be again associated with effects of random impurities.

\section{B) The case of $s=50 \mathrm{~nm}$ as in A:}

One may expect that the drastic fluctuations in density profiles, self-consistent potentials and conductance patterns discussed above should be reduced by an increase in a distance between the donor layer and 2DEG region. This is clearly supported by the numerical results shown in Figs. 7 and 8 for the case of $s=50$ $\mathrm{nm}$. The smeared density profiles in Figs. 7a-c correspond to conductance in the vicinity of $G=0.27 G_{0}, G=0.62 G_{0}$ and $G=0.8 G_{0}$. From the spin polarization picture drawn in Figs. 8a-c one can see that the region of non-zero spin-polarization in the case when $G=0.27 G_{0}$ forms a stripe in the middle of the QPC (Fig. 8a) and splits in two (Fig. 8b) and three (Fig. 8c) rows when $G=0.62 G_{0}$ and $G=0.8 G_{0}$, respectively. As a whole, the conductance behavior in the case $s=50 \mathrm{~nm}$ resembles the usual conductance for a perfect QPC free of resonances, that is, the transport turns to be ballistic though the fluctuations of density and potentials are still present.

\section{C) The case of $s=100 \mathrm{~nm}$ as in A:}

In this case the confinement is essentially much more shallow than in the preceding cases $\mathbf{A}$ and $\mathbf{B}$. This immediately results in the fact that the electron density and spin polarization in Figs. 9 and 10 are almost free from fluctuations, and the conductance displayed in Fig. 11 contains both the usual conductance step at $G_{0}$ and the 0.7 anomaly. Note that Figures 9 and 10 are drawn for the same set of splitgate voltages which correspond to the following conductance curves: a) $G=0.3 G_{0}$ and b) $G=0.7 G_{0}$ for $V_{s g}=-0.65 \mathrm{eV}$ and $V_{s g}=-0.52 \mathrm{eV}$ correspondingly. A remarkable feature is the presence of an extended region of spin polarization in the middle of a QPC in Fig. 10a which gives rise to a weak anomaly at $G=0.3 G_{0}$ in the conductance curves in Fig. 11. There are also two stripes of spin polarization for the case in Fig. 10b which corresponds to conductance $G=0.7 G_{0}$. In the case $G=0.85 G_{0}$ (not shown here) the third row is formed similar to case $\mathbf{B}$. The 0.7 conductance anomaly is evidently accompanied by the formation of parallel polarized stripes. The wider the $\mathrm{QPC}$ is, the larger is the number of stripes, here from 2 to 3 . We recall that for a more narrow QPC there is only one polarized stripe of this kind at $0.7[9]-[12]$.

\section{D) The case of partially ionized donors and short range order}

Cases A-C refer to uncorrelated fully ionized donors. This assumption may give rise to strong fluctuations at moderate values of $s$ that appear to be too strong as in [36]. However, in GaAs/AlGaAs heterostructures donor electrons in the doping layer may remain in shallow states or be trapped in deep DX centers. Thus one 
should expect correlation among the charged donors [48]-[49] that should weaken the fluctuations in conductance. In a final series of our numerical experiments we have studied a number of structures with the donor density $n_{i}=1.4 \cdot 10^{17} \mathrm{~cm}^{-3}$ which is effectively four times less than in the previous cases $\mathbf{A}-\mathbf{C}$. This case can be realized when only a fraction of the donors are ionized. Confinement and total potentials as well as electron density then become less randomized (as it is seen from Fig. 12), and in accordance with this our calculated conductance curve is more smooth (Fig. 13). Indeed, in most of experiments conductance through a QPC is free of any additional resonances. Conversely, if this is the case, we can conclude that not all donors are ionized and thus contribute to the formation of confinement potentials in a given realistic structure.

In effect, in the system of partially ionized donors they should have a tendency to partial short range order, i.e., more or less ordered donor distribution within a shorter range. For better understanding of physics processes involved we have calculated a pair correlation function given by [55]:

$$
g(r)=\frac{n(r)}{4 \pi r^{2} \Delta r N n_{i}}
$$

where $\mathrm{n}(\mathrm{r})$ is the number of donors laying within the interval $\Delta r=10 \mathrm{~nm}$ around the position $r$ which is a random number designating the position of donors within the doping layer, $\mathrm{N}$ is a total number of donors (800 in this case). The result of this calculation is demonstrated by Fig. 14 where $g(r)$ is plotted in the form of a histogram. This histogram has a maximum at short distances and approaches to one for long distances between donors. In other words, the fluctuations in such system remind of the pair correlation function for liquids [55].

\section{Concluding remarks}

We have investigated the role of randomly distributed impurities on electron transport in QPCs made from a modulation-doped semiconductor heterostructure. The results of our simulations show that the disorder may have a strong influence on the electron transport in such systems if the donor layer is situated at distances less than $50 \mathrm{~nm}$ from the 2DEG. There are two main effects caused by disorder: a) occurrence of new resonances, and b) smoothing of the usual plateaux in conductance patterns.

Our studies have been performed for the two geometries of the gates, the first one with only split gates, and the other one with an additional top gate situated over the split gates. In the latter case there is a possibility to vary electron density within a fixed confinement which gives an opportunity to separate the effects on conductance caused by impurities from electron-electron interactions in a more distinct way. For example, in [31] the top gate voltage $V_{t g}$ was applied and swept while the split gates were loaded by fixed voltage $V_{s g}$. In this case the resonances in the conductance are shifted for different $V_{s g}$ which indicates that they may be caused by disorder effects. A similar effect is seen in Figure 5. In asymmetric quantum point contacts created by applying the off-set voltage to the two split gates (Figure 6) there are feeble resonances which eventually disappear. The strength of the resonances depends on the value of off-set voltage $\Delta V_{s g}$ : the larger $\Delta V_{s g}$ the cleaner 
conductance characteristics can be reached. We also note that appearance of double and triple stripes in Figs. 8 and 10.

Naturally, both for device applications and for studying effects of pure electronelectron interaction one would like to diminish the effects of disorder. One of the possible ways can be realized by using shallow undoped AlGaAs/GaAs heterostructure [57]-[58]. It turns out, however, that even in this case scattering from remote surface charges can affect electron transport and reduce a quantum lifetime. In particular, the electrons of a very high mobility have been clearly identified in the system with the 2DEG depth exceeds $200 \mathrm{~nm}$. The role of background impurities in the electronic properties of undoped heterostructure of this type has been investigated in [59]. It has been shown that both $\mathrm{C}$ background impurities and Si DX centers contribute to the disorder that can be altered by optical illumination and influence the conductance. Further, the new method of multi-tip scanning gate microscopy has been proposed for studying of ballistic transport in 2DEG [60]. This so-called SGM technique can be used for mapping the potential defects in the conducting channel by resolving resonances caused by them.

Our final remark concerns the quasi-bound states which are inherent both in symmetrically and asymmetrically charged QPCs. As argued in [61], the quasi-bound states in asymmetric QPCs may be formed because of a momentum mismatch (the same conclusion has been stated in more recent paper [56]). That should give rise to experimentally observed resonances on conductance. However, confinement potentials in realistic structures are much softer than in a structure with a model rectangular potential used in [61]. Such soft potentials can hardly produce resonances and quasi-bound states as was discussed in [27]. From the other side, the distance between donor layer and 2DEG in [61] is about $20 \mathrm{~nm}$ that belongs, in accordance with the data of our simulation, to the interval potentially dangerous for the appearance of resonances due to the quasi-bound states caused by disorder effects. This also applies to [34] in which the distance is $40 \mathrm{~nm}$. In such cases the length of the channels becomes undefined, in particularly close to pinch-off (as in Fig. 3a). In this respect, one should recall the weakening of the effects of disorder and the possible existence of a short range order in partly ionized random donors distributions.

\section{Aknowledgements}

Authors appreciate Carl Trygger Science Foundation of Sweden for the financial support (project number CTS: 13-519). Discussions with M. Pepper and K. J. Thomas, University College, London, L. W. Smith, when at Cavendish Laboratory, Cambridge, and A.M. Chang, Duke University are greatfully acknowledged. We are grateful for support from National Supercomputer Centre at Linkping University, Sweden.

\section{Figure captions}

Figure 1: Schematic view of the split-gate device with randomly distributed donors (left panel) and top view of the device with geometry of the gates (right panel); $\mathrm{s}$ is the distance between 2DEG and the random donors layer. $V_{g}$ is the voltage to the two semi-infinite metallic gates and is used to define the left and right electron resevoirs; the two metallic finger gates with voltage $V_{s g}$ are used to regulate the QPC potential in conjuction with the metallic top gate at voltage $V_{t g}$, the computation area is $400 \mathrm{x} 400 \mathrm{~nm}^{2}$. 
Figure 2 (color on line): a) Confinement potential in the case of $\mathrm{s}=10 \mathrm{~nm}$ and $\left.V_{s g}=-1.6 \mathrm{eV} ; \mathrm{b}\right)$ total electrostatic potentials in the case of $\mathrm{s}=10 \mathrm{~nm}$ and $V_{s g}=$ $-1.6 \mathrm{eV}, \mathrm{c})$ total electrostatic potentials in the case of $\mathrm{s}=10 \mathrm{~nm}$ and $V_{s g}=-1 \mathrm{eV}$.

Figure 3 (color on line): Total electron densities in the case of $\mathrm{s}=10 \mathrm{~nm}$ and a) $\left.\left.V_{s g}=-1.6 \mathrm{eV}, \mathrm{b}\right) V_{s g}=-1.4 \mathrm{eV}, \mathrm{c}\right) V_{s g}=-1 \mathrm{eV}$.

Figure 4 (color on line): Conductance $G$ as a function of split-gate potential $V_{s g}$ in the case of $\mathrm{s}=10 \mathrm{~nm}$ : a) total conductance; b) conductance for up-spin electrons; c) conductance for down-spin electrons.

Figure 5 (color on line): Total conductance $G$ as a function of top gate potentials $V_{t g}$ in the case of $\mathrm{s}=10 \mathrm{~nm}$ and a) $\left.\left.V_{s g}=-0.8 \mathrm{eV}, \mathrm{b}\right) V_{s g}=-1 \mathrm{eV}, \mathrm{c}\right) V_{s g}=-1.2$ $\mathrm{eV}$.

Figure 6 (color on line): Total conductance $G$ as a function of top gate potentials $V_{t g}$ in the case of $\mathrm{s}=10 \mathrm{~nm}$ and a) $\left.V_{s g 1}=-1 \mathrm{eV}, V_{s g 2}=-1 \mathrm{eV}, \mathrm{b}\right) V_{s g 1}=-1 \mathrm{eV}$ $V_{s g 2}=-1.2 \mathrm{eV}$ and c) $V_{s g 1}=-1.2 \mathrm{eV}$ and $V_{s g 2}=-1 \mathrm{eV}$.

Figure 7 (color on line): Total electron densities in the case of $\mathrm{s}=50 \mathrm{~nm}, V_{t g}=0$ $\mathrm{eV}$ and a) $\left.\left.V_{s g}=-0.98 \mathrm{eV}, \mathrm{b}\right) V_{s g}=-0.75 \mathrm{eV}, \mathrm{c}\right) V_{s g}=-0.65 \mathrm{eV}$.

Figure 8 (color on line): Spin polarization in the case of $\mathrm{s}=50 \mathrm{~nm}, V_{t g}=0 \mathrm{eV}$ and a) $\left.V_{s g}=-0.98 \mathrm{eV}, \mathrm{b}\right) V_{s g}=-0.75 \mathrm{eV}$, c) $V_{s g}=-0.65 \mathrm{eV}$.

Figure 9 (color on line): Total electron densities in the case of $\mathrm{s}=100 \mathrm{~nm}, V_{t g}=0$ $\mathrm{eV}$ and a) $\left.V_{s g}=-0.65 \mathrm{eV}, \mathrm{b}\right) V_{s g}=-0.52 \mathrm{eV}$.

Figure 10 (color on line): Spin polarization in the case of $\mathrm{s}=100 \mathrm{~nm}, V_{t g}=0 \mathrm{eV}$ and a) $\left.V_{s g}=-0.65 \mathrm{eV}, \mathrm{b}\right) V_{s g}=-0.52 \mathrm{eV}$.

Figure 11 (color on line): Conductance $G$ as a function of split gates potentials $V_{s g}$ in the case of $\mathrm{s}=100 \mathrm{~nm}$ and $V_{t g}=0 \mathrm{eV}$ : a) total conductance; b) conductance for up-spin electrons; c) conductance for down-spin electrons.

Figure 12 (color on line): a) Confinement potential and b) electron density in the case of $\mathrm{s}=10 \mathrm{~nm}$ and $V_{s g}=-1.6 \mathrm{eV}$, and donor density: $n_{i}=1.4 \cdot 10^{17} \mathrm{~cm}^{-3}$.

Figure 13 (color on line): Conductance $G$ as a function of split gates potentials $V_{s g}$ in the case of $\mathrm{s}=10 \mathrm{~nm}, V_{t g}=0 \mathrm{eV}$ and donor density: $n_{i}=1.4 \cdot 10^{17} \mathrm{~cm}^{-3}$ : a) total conductance; b) conductance for up-spin electrons; c) conductance for downspin electrons.

Figure 14: Pair correlation function for the case of donor density: $n_{i}=1.4$. $10^{17} \mathrm{~cm}^{-3}$.

\section{REFERENCES}

1. Berggren K-F and Pepper M Phil. Trans. Roy. Soc. A 20103681141.

2. Pepper M and Bird J, (eds.) J. Phys.: Cond. Matter 200820 No.16 (Special section: "The 0.7 feature and interactions in one-dimentional systems", and references therein).

3. Ferry D K, Goodnick S M and Bird J P "Transport in Nanostructures" 2010 2nd ed. Cambridge.

4. Micolich A P J. Phys.: Condens. Matter 201123443201.

5. Thomas K J, Nicholls J T, Simmons M Y, Pepper M, Mace D R, and Ritchie D A Phys. Rev. Lett. 199677135. 
6. Thomas K J, Nicholls J T, Appleyard N J, Simmons M Y, Pepper M, Mace D R, Tribe W R, and Ritchie D A Phys. Rev. B 1998584846.

7. Wang C-K and Berggren K-F Phys. Rev. B 199654 14257; ibid. 199857 4552 .

8. Bychkov A M, Yakimenko I I and Berggren K-F Nanotechnology 2000 11, 318.

9. Berggren K-F and Yakimenko I I Phys. Rev. B 2002 66, 085323.

10. Starikov A A, Berggren K-F, and Yakimenko I I Phys. Rev. B 200367235319.

11. Jaksch P, Yakimenko I I, and Berggren K-F Phys. Rev. B 200674235320.

12. Berggren K-F and Yakimenko I I, J. Phys.: Condens. Matter 200820164203.

13. Szafran B, Nowak M P, Wach E, and Zebrovski D P Phys. Lett. A 2014378 1036 .

14. Hirose K, Meir Y, and Wingreen N S Phys. Rev. Lett. 200390026804.

15. Rejec T and Meir Y Nature 2006442900.

16. Cronenwett S M, Lynch H J, Goldhaber-Gordon D, Kouwenhoven L P, Marcus C M, Hirose K, Wingren N S and Umansky V Phys. Rev. Lett. 200288 226805 .

17. Song T and Ahn K-H Phys. Rev. Lett 2011106057203.

18. Yoon Y, Mourokh L, Morimoto T, Aoki N, Ochiai Y, Reno J L, Bird J P, Phys. Rev. Lett. 200799136805.

19. Y. Ren Y, Yu W W, Frolov, S M J, Folk J A, and W. Wegscheider W Phys. Rev. B 201082045313.

20. Iqbal M J, Levi Roi, Koop E J, Dekker J B, de Jong J P, van der Velde H M, Reuter D, Wieck A D, Aguado R, Meir Y, van der Wal C H Nature 2013501 79 .

21. Bauer F, Heyder J, Schubert E, Borowsky D, Taubert D, Bruognolo B, Schuh D, Wegscheider W, von Delft J, and Ludwig S Nature 201350173.

22. Hew W K, Thomas K J, Pepper M, Farrer I, Anderson D, Jones G A C, Ritchie D A Phys. Rev. Lett. 2009102056804.

23. Kumar S, Thomas K J, Smith L W, Pepper M, Ceeth G L, Farrer I, Ritchie D, Jones G and Griffiths G Phys. Rev. B $201490201304(\mathrm{R})$.

24. Güclü A D, Umrigar C J, Jiang H, Baranger H J Phys. Rev. B 200980 201302(R).

25. Mehta A C, Umrigar C J, Meyer J S, and Baranger H J Phys. Rev. Lett. 2013 110, 246802. 
26. Welander E, Berggren K-F, Yakimenko I I Phys. Rev. B 2010 82, 073307.

27. Yakimenko I I, Tsykunov V S and Berggren K-F J. Phys. Condens. Matter 201325072201.

28. Williamson J G, Timmering C E, Harmans C J P, J.J Harris J J, Foxon C T Phys. Rev. B 1990427675 .

29. Timp G in Semiconductors and Semimetals, eds. Reed M A 1992 New York Academic Press 113.

30. Stopa M Phys. Rev. B 1996539595.

31. Smith L W, Thomas K J, Pepper M, Ritchie D A, Farrer I, Griffiths G P, Jones G A C J. of Phys.: Conf. Series 2012376012018.

32. Yang Q-Z, Kelly M J, Farrer I, Beere H E, and Jones G A C Appl. Phys. Lett. 2009 94, 033502.

33. Smith L W, Al-Taie H, Sfigakis F, See P, Lesage A A J, Xu B, Griffiths J P, Beere H E, Jones G A C, Ritchie D A, Kelly M J, and Smith C G Phys. Rev. B 201490045426.

34. Smith L W, Al-Taie H, Lesage A A J, Sfigakis F, See P, Griffiths J P, Farrer I, Jones G A C, Ritchie D A, Kelly M J, and Smith C G Phys. Rev. B 2015 91, 235402 .

35. Nixon J A and Davies J H Phys. Rev. B 1990417929.

36. Nixon J A, Davies J H, Baranger H U Phys. Rev. B 19914312638.

37. Zagoskin A M, Rashkeev S N, Shekhter R I, Wendin G J. Phys.: Condens. Matter 199576253.

38. Aoki N, da Cunha C R, Akis R, Ferry D, Ochiai Y Appl. Phys. Lett. 200587 225501 .

39. da Cunha C R, Aoki N, Morimoto T, Ochiai Y, Akis R, and Ferry D Appl. Phys. Lett. 200689242109.

40. Debray P, Rahman S M S, Wan J, Newrock R S, Cahay M, Ngo A T, Ulloa S E, S T Herbert, Muchammad M,and Johnson M Nature Nanotechnology 2009 4759 .

41. Wan J, Cahay M, Debray P, and Newrock R S Phys. Rev. B 200980155440.

42. Wan J, Cahay M, Debray P, and Newrock R S J. Nanoelectron. Optoelectron. 2011695.

43. Das P P, Bhandari N, Wan J, Charles J, Cahay M, Chetry K B, Newrock R S, and Herbert S T Nanotechnology 201223215201.

44. Liu B and Heller E J Phys. Rev. Lett. 2013111236804. 
45. Chuang P, Ho Sh-Ch, Smith L W, Sfigakis F, Pepper M, Chen Ch-H, Fan J-Ch, Griffiths J P, Farrer I, Beere H E, Jones G A C, Ritchie D A, and Chen T-M Nature Nanotechnology 2015103539.

46. Datta S and Das B Appl. Phys. Lett. 199056665.

47. Cahay M Nature Nanotechnology 201510 (1) 21.

48. Buks E, Heiblum R M Phys. Rev. B 19944914790.

49. Shikler R, Heiblum R M, and Umanski V Phys. Rev. B 19975515427.

50. Davies J H. The Physics of Low-Dimensional Semiconductors: An Introduction 1998 Cambridge University Press, Cambridge.

51. Martorell J, Wu H, and Sprung D W L Phys. Rev. B 19945017298.

52. Ji Zh-Li and Berggren K-F Phys. Rev. B 199552 R11 607.

53. Tanatar B, Ceperley D M Phys. Rev. B 1989395005.

54. Lassl A, Schlagheck P, and Richter K Phys. Rev. B 200775045346.

55. Hansen J P and McDonald I R Theory of Simple Liquids 2005 Academic Press.

56. Zhang H, Wu Ph M, Chang A M Phys. Rev. B 201388075311.

57. Laroche D, Das Sarma S, Gervais G, Lilly M P, and Reno J R Appl. Phys. Lett. 201096162112.

58. Wang D Q, Chen J C H, Klochan O, Das Gupta K, Reuter D, Wieck A D, Ritchie D A and Hamilton A R Phys. Rev. B 201387195313.

59. See A M, Hamilton A R, Micolich A P, Aagesen M, and Lindelof P E Phys. Rev. B 201591085417.

60. Kolasinski K, Szafran B and Hackens B Phys. Rev. B 201591205314.

61. Wu Ph M, Li P, Zhang H and Chang A M Phys. Rev. B 201285085305. 


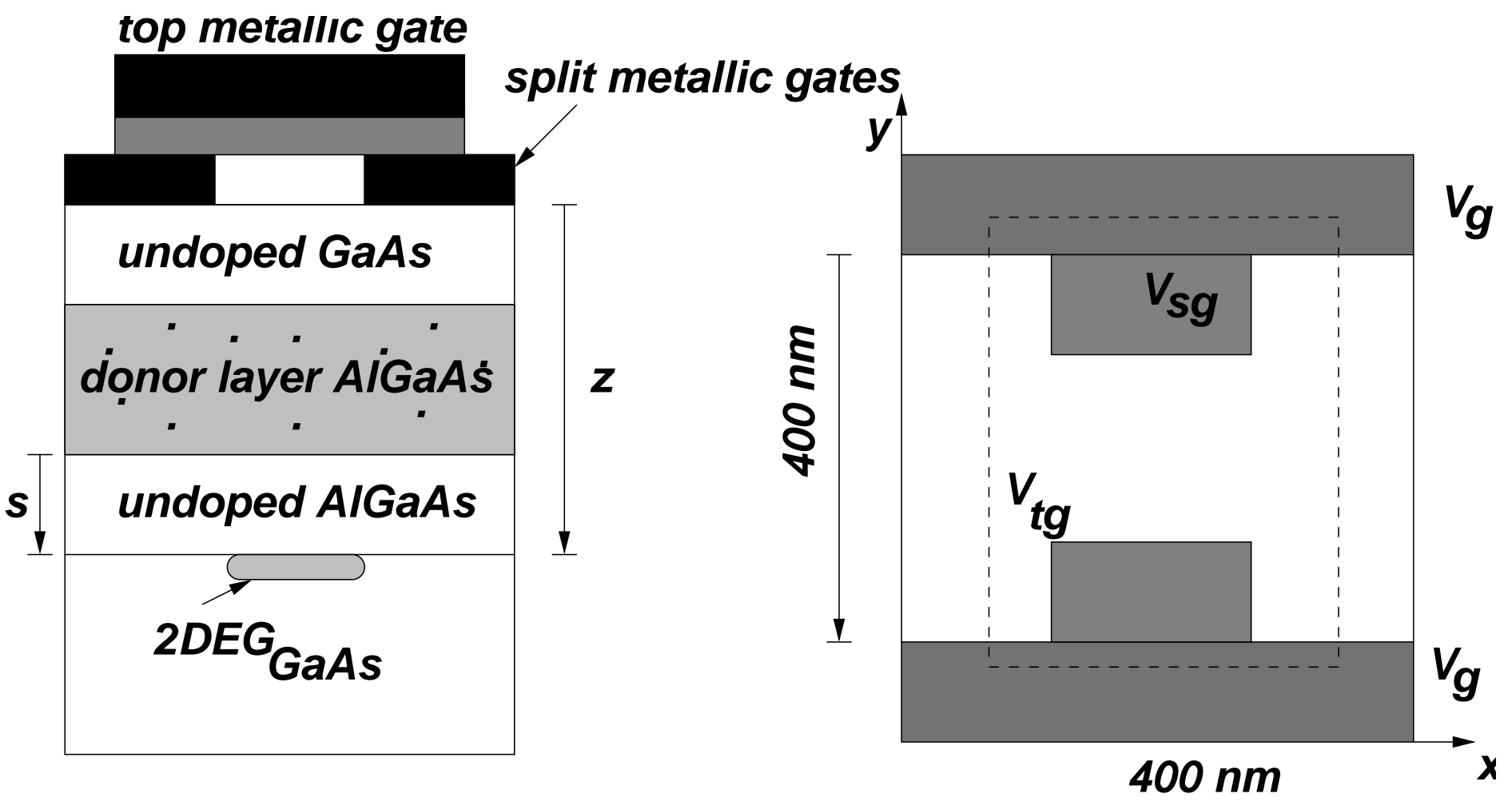

a)

b) 


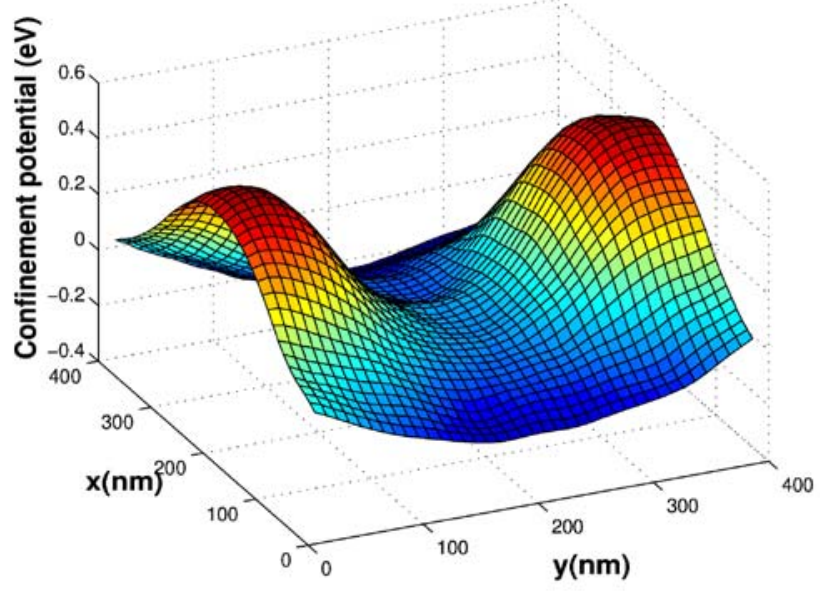

a)

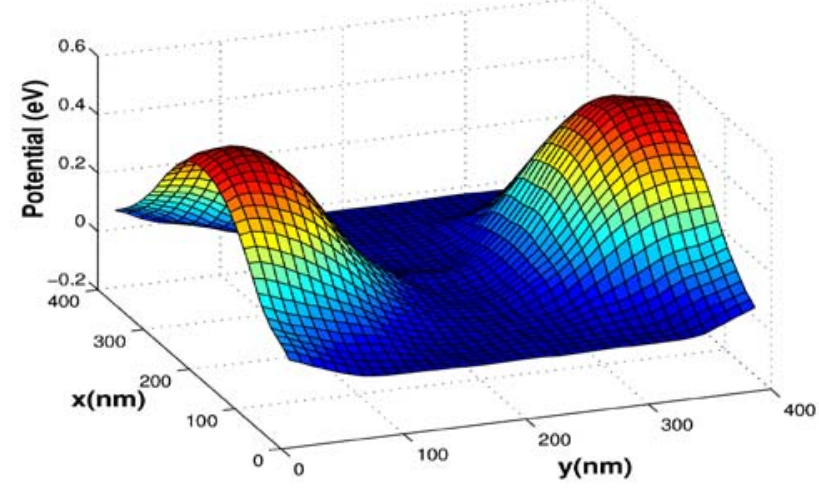

b)

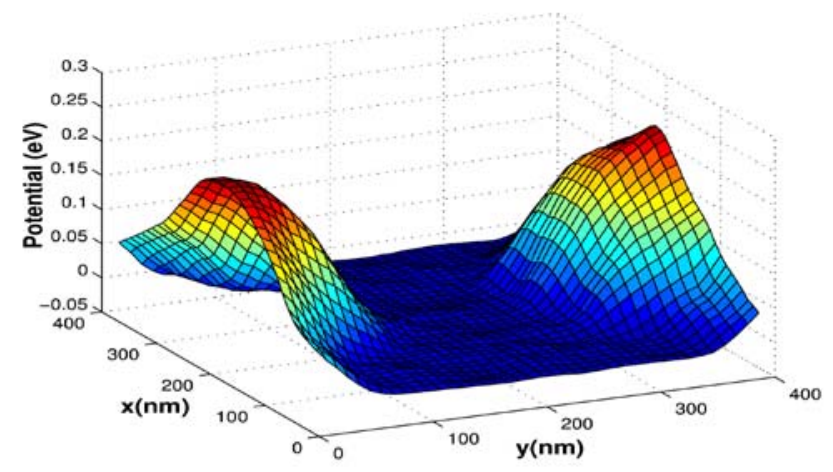

c) 


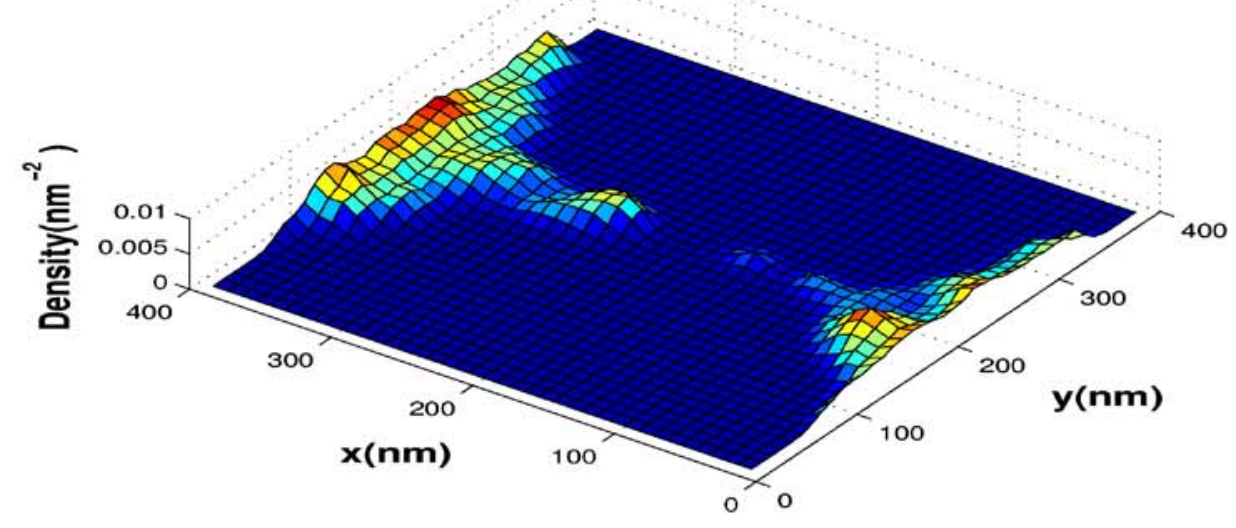

a)

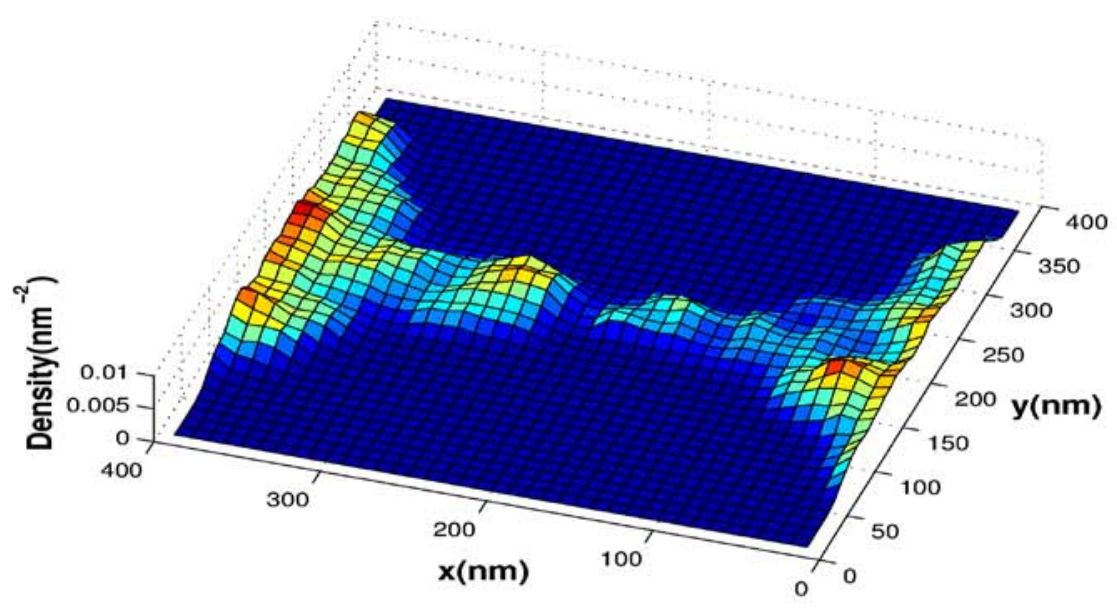

b)

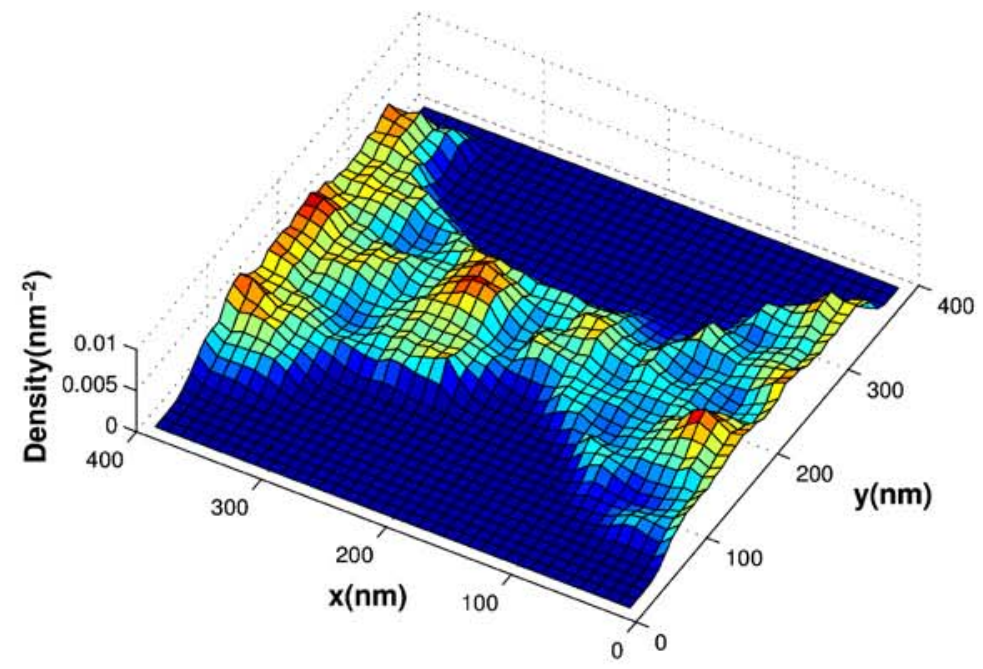

c) 


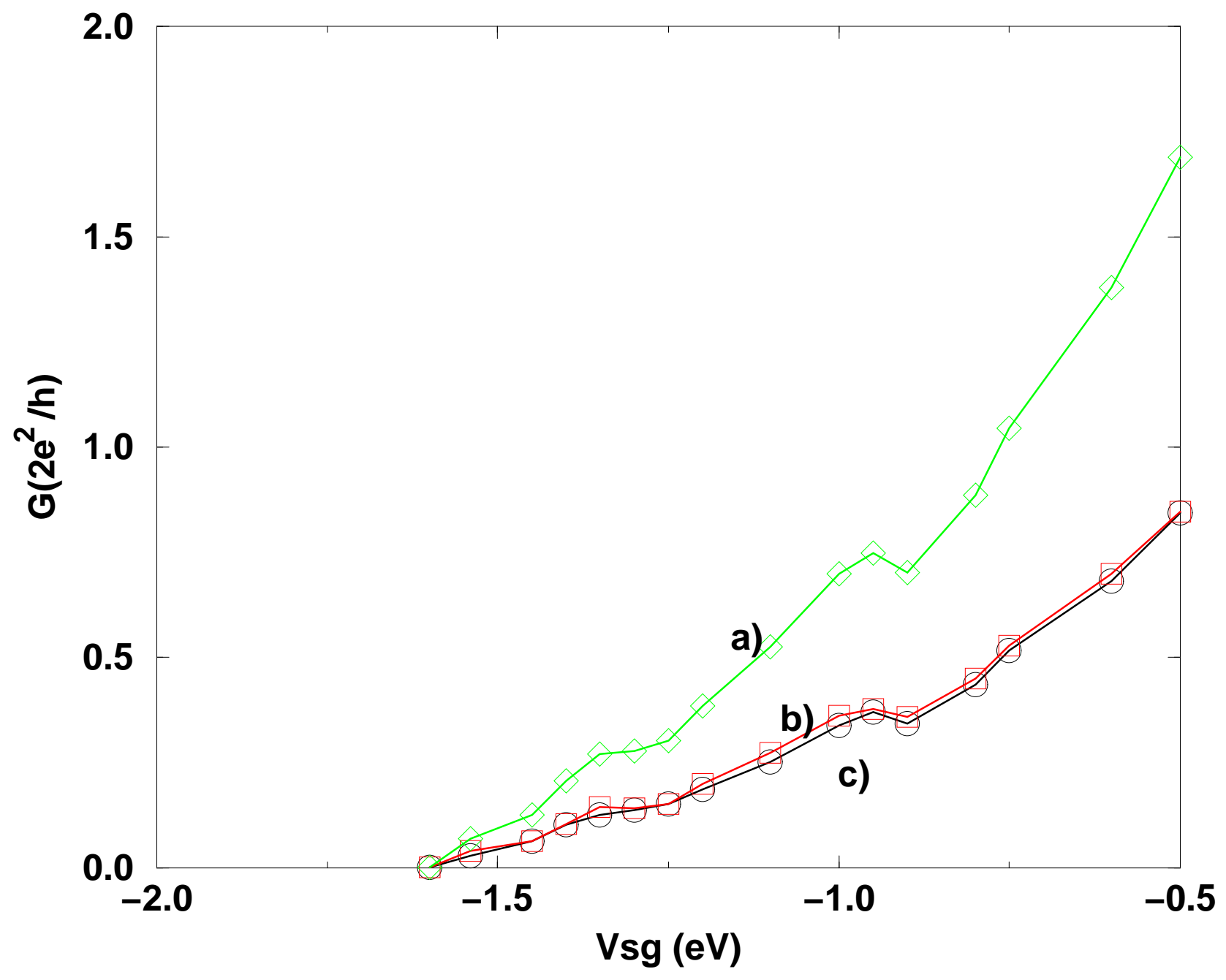




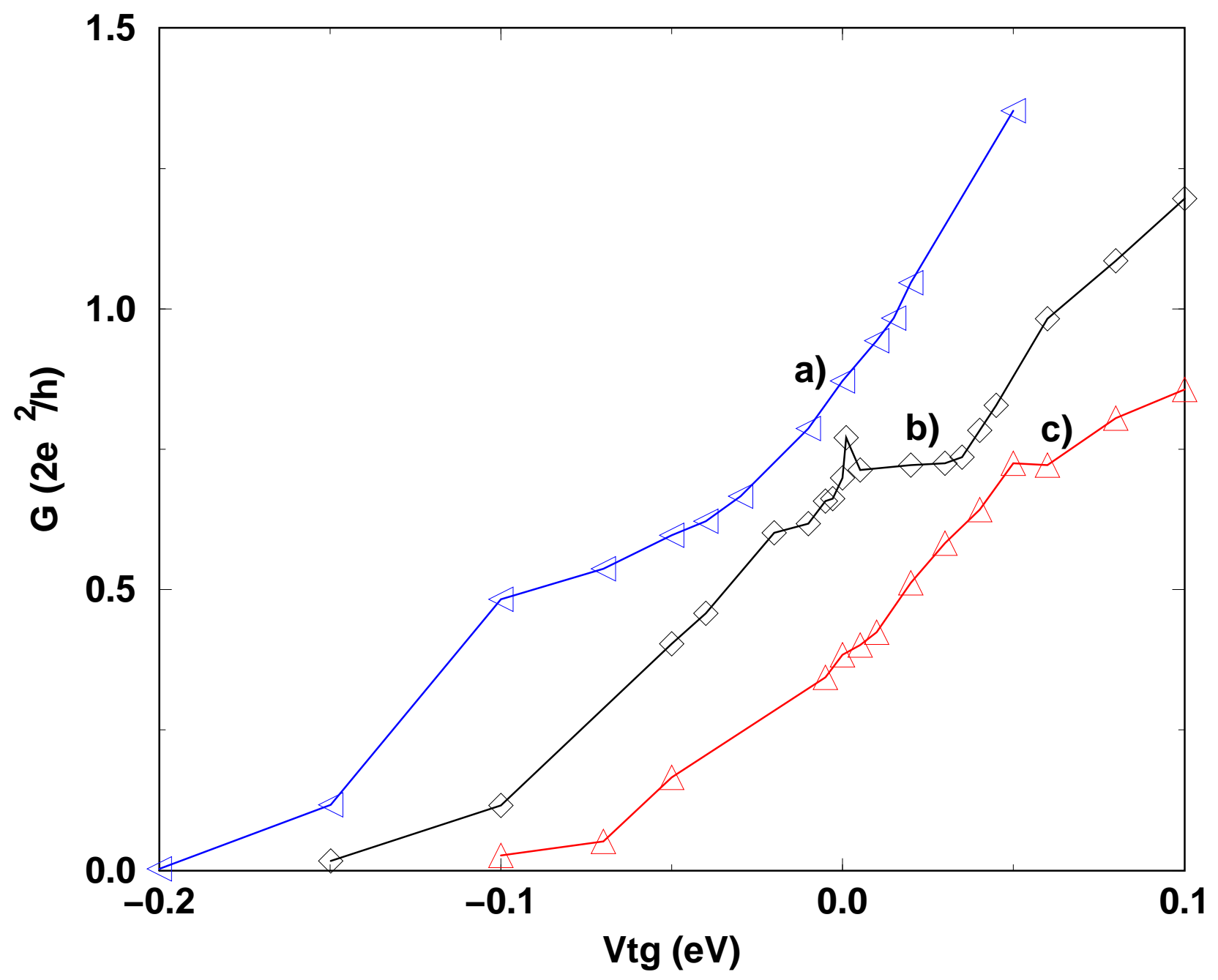




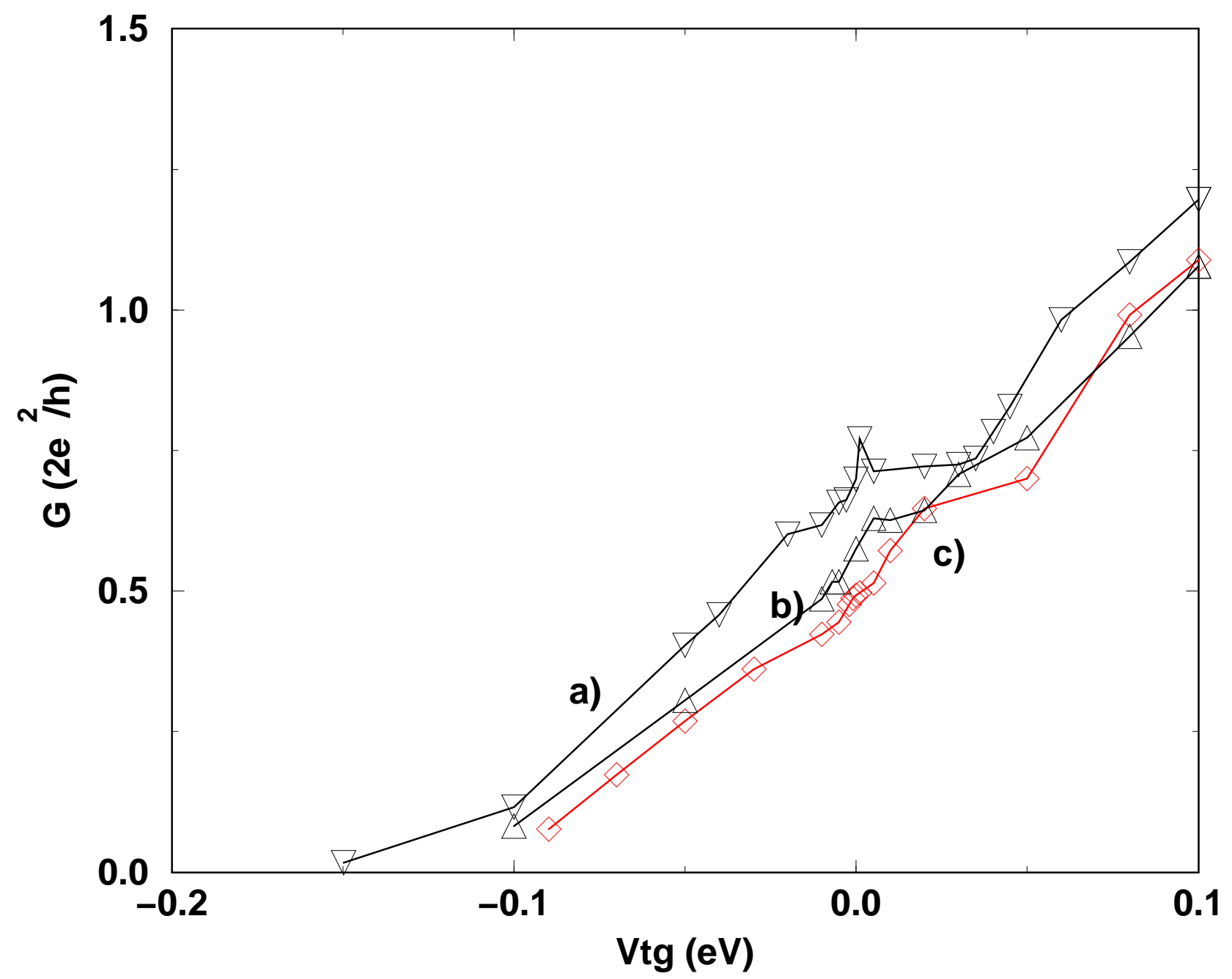




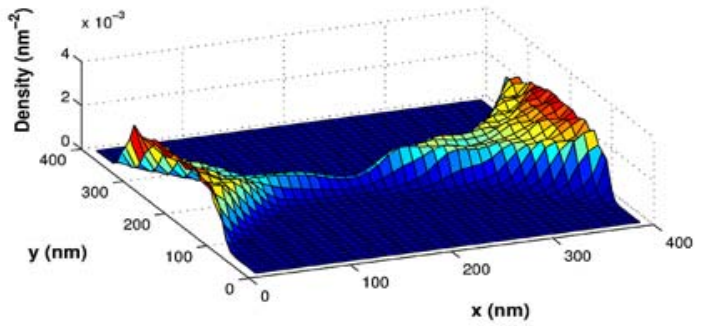

a)

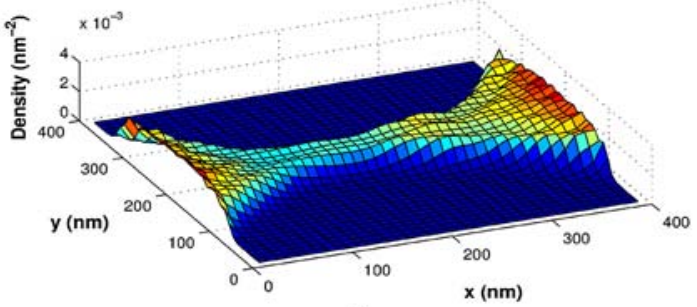

b)

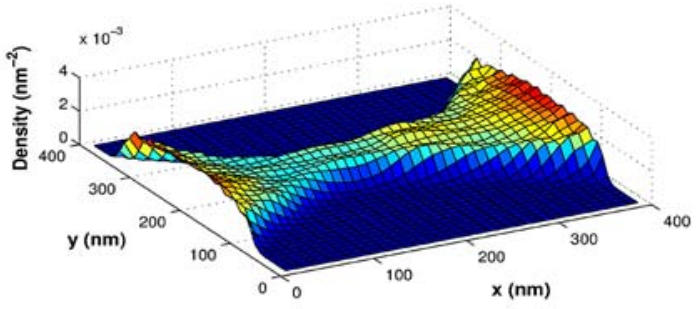

c) 


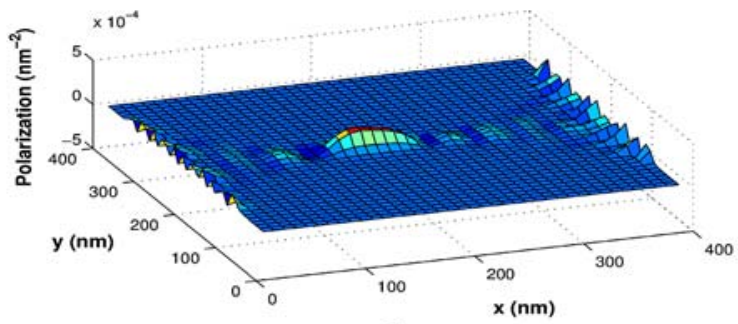

a)

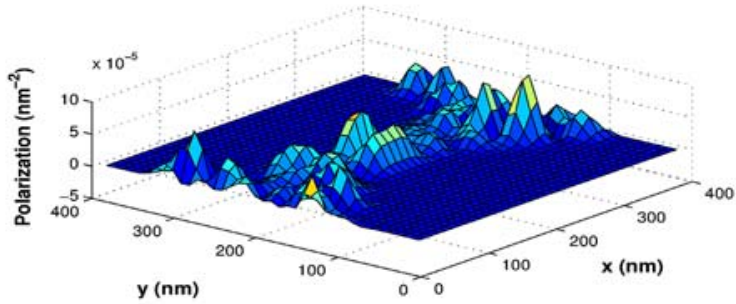

b)

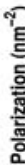

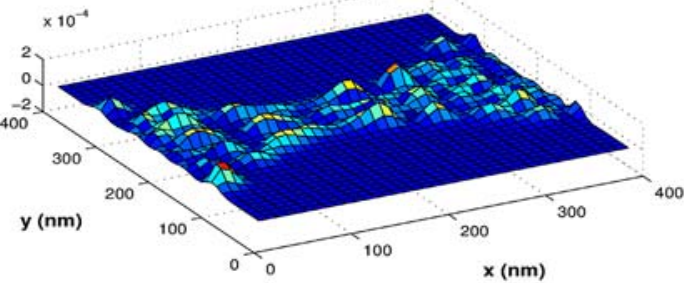

c) 
$\times 10^{-3}$

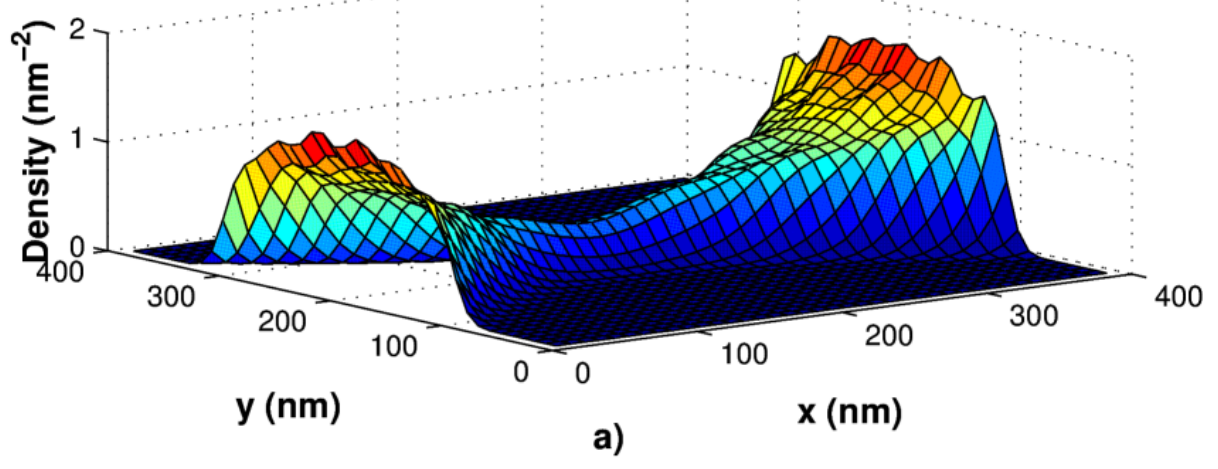

$\times 10^{-3}$

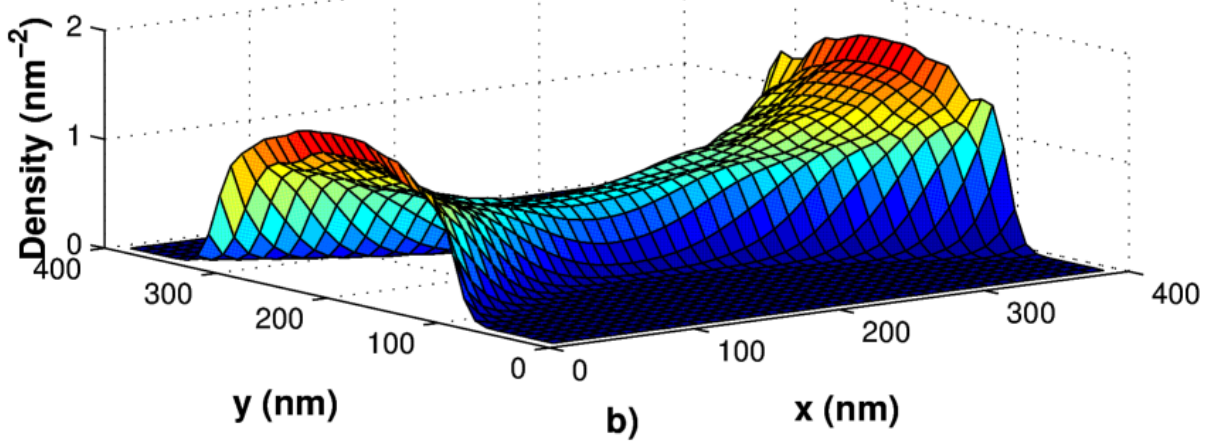



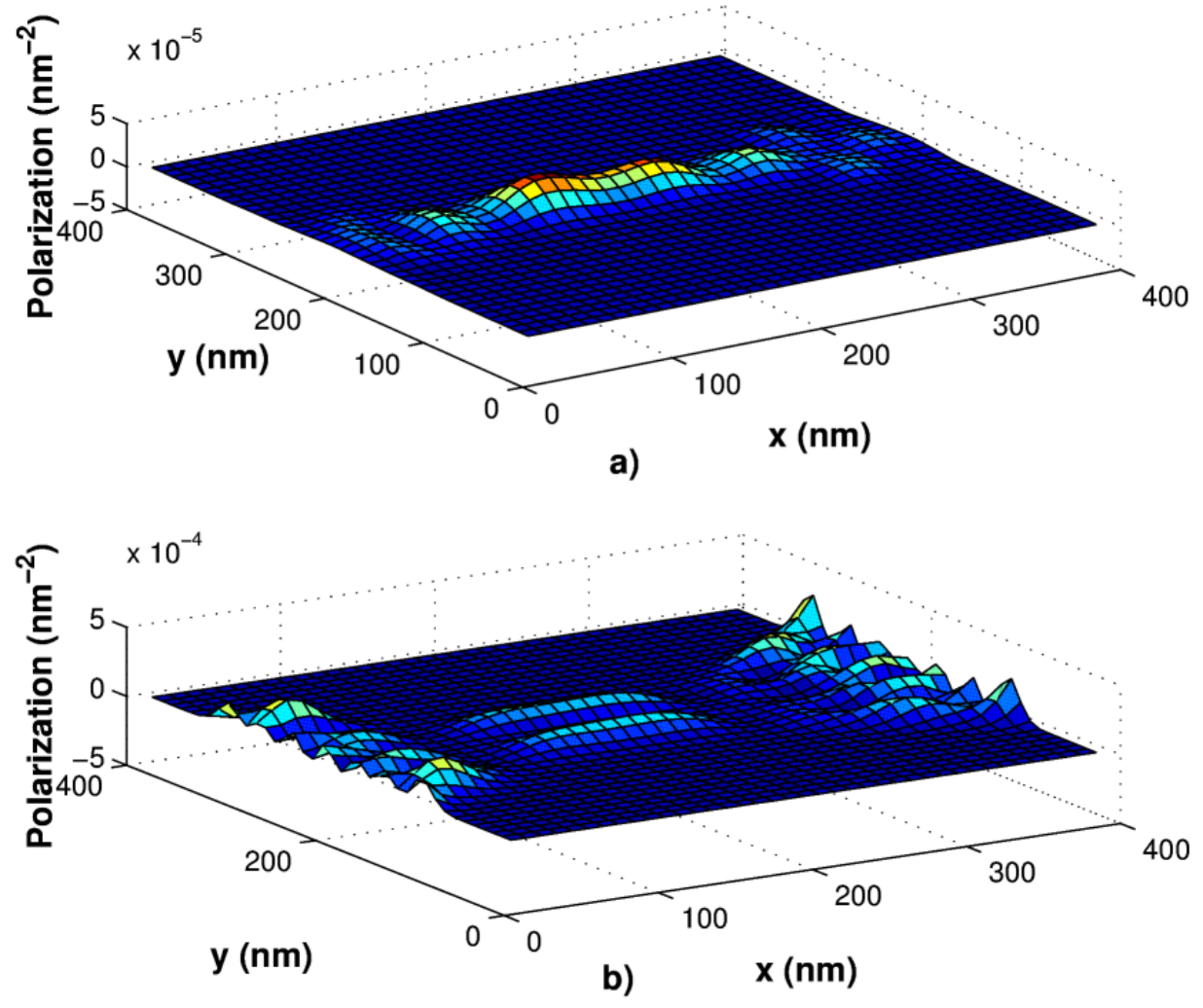


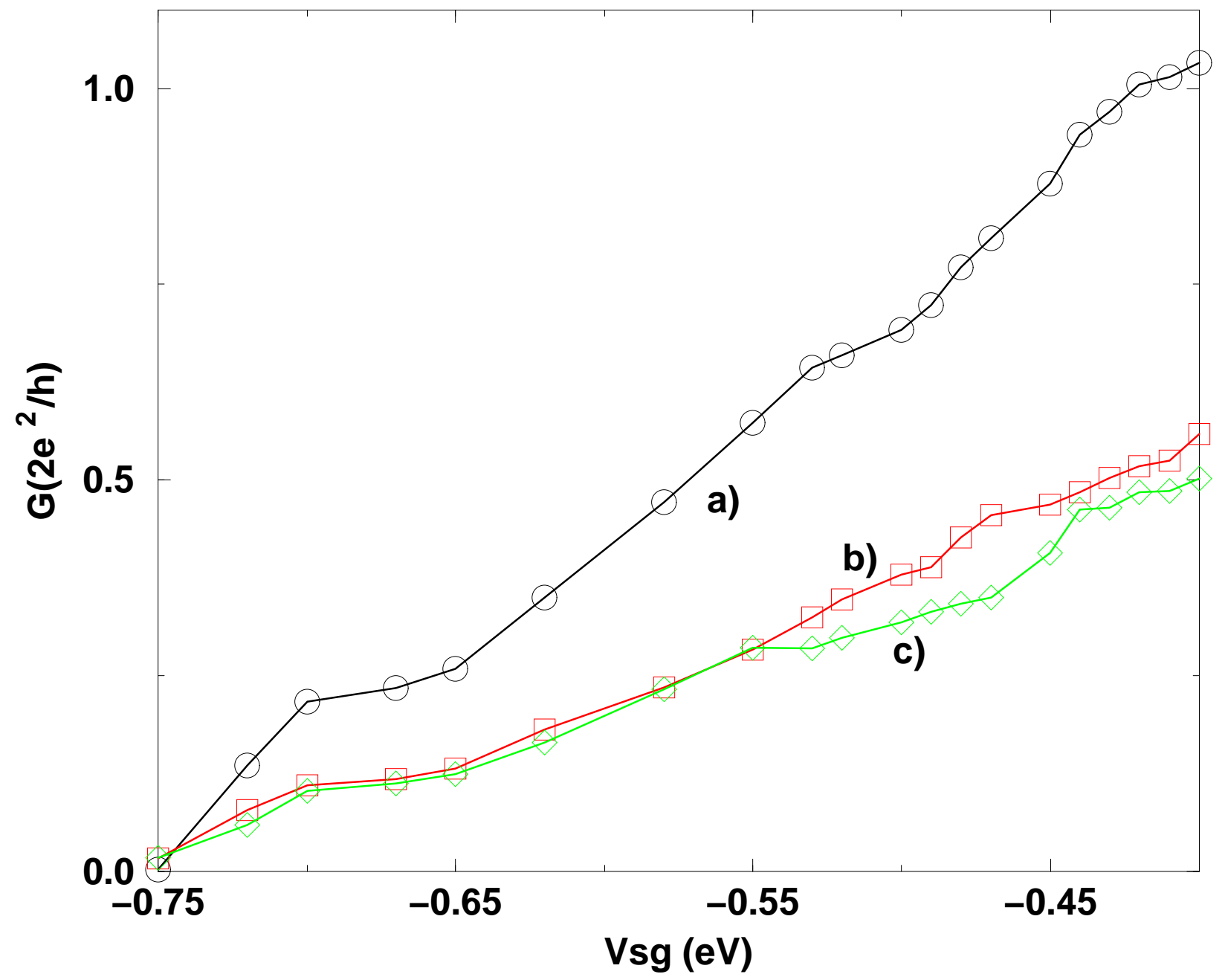




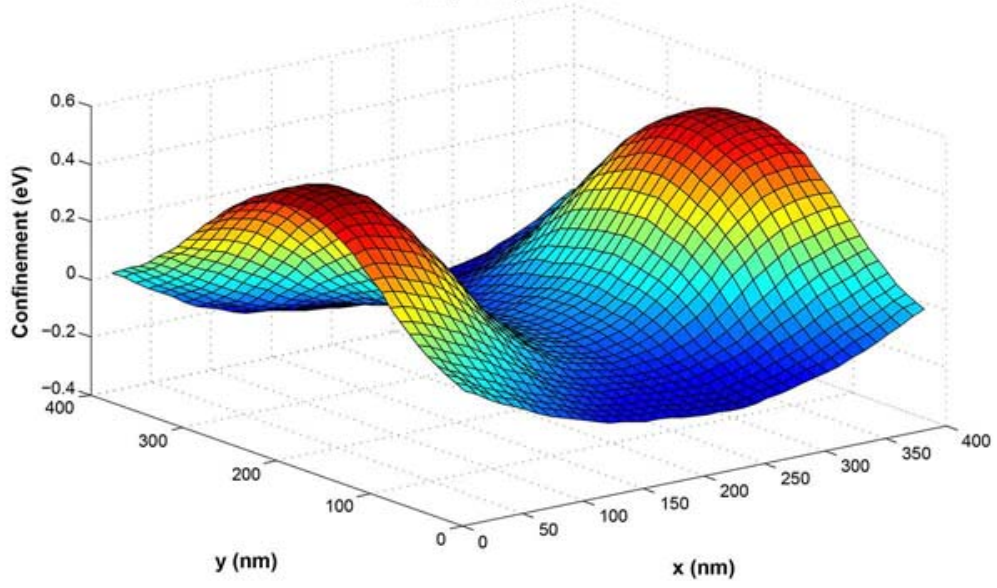

a)

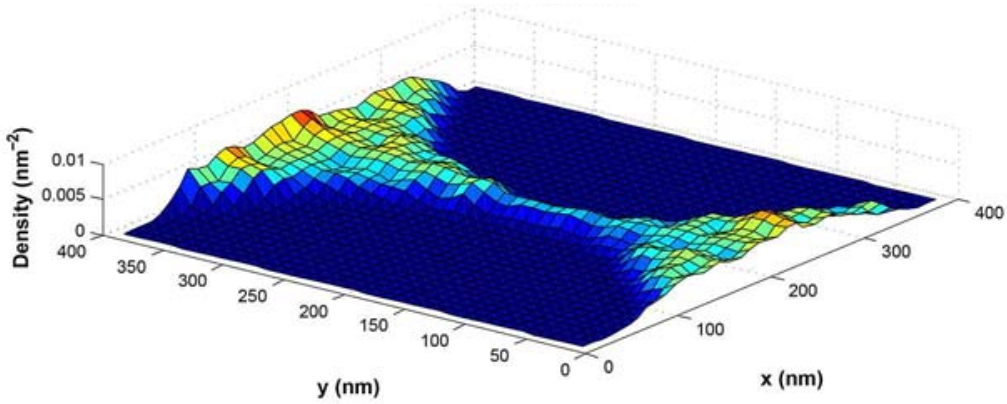

b) 


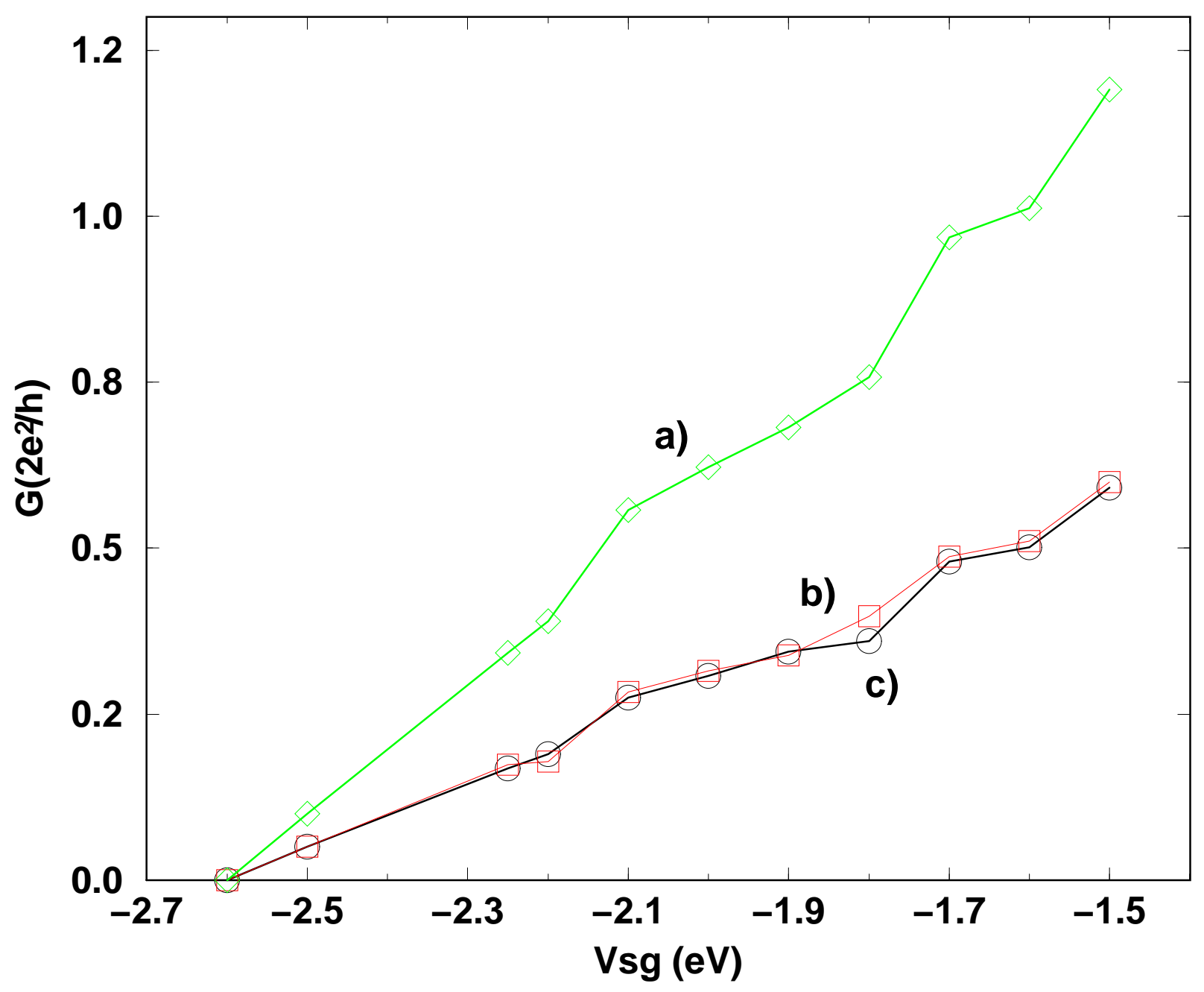




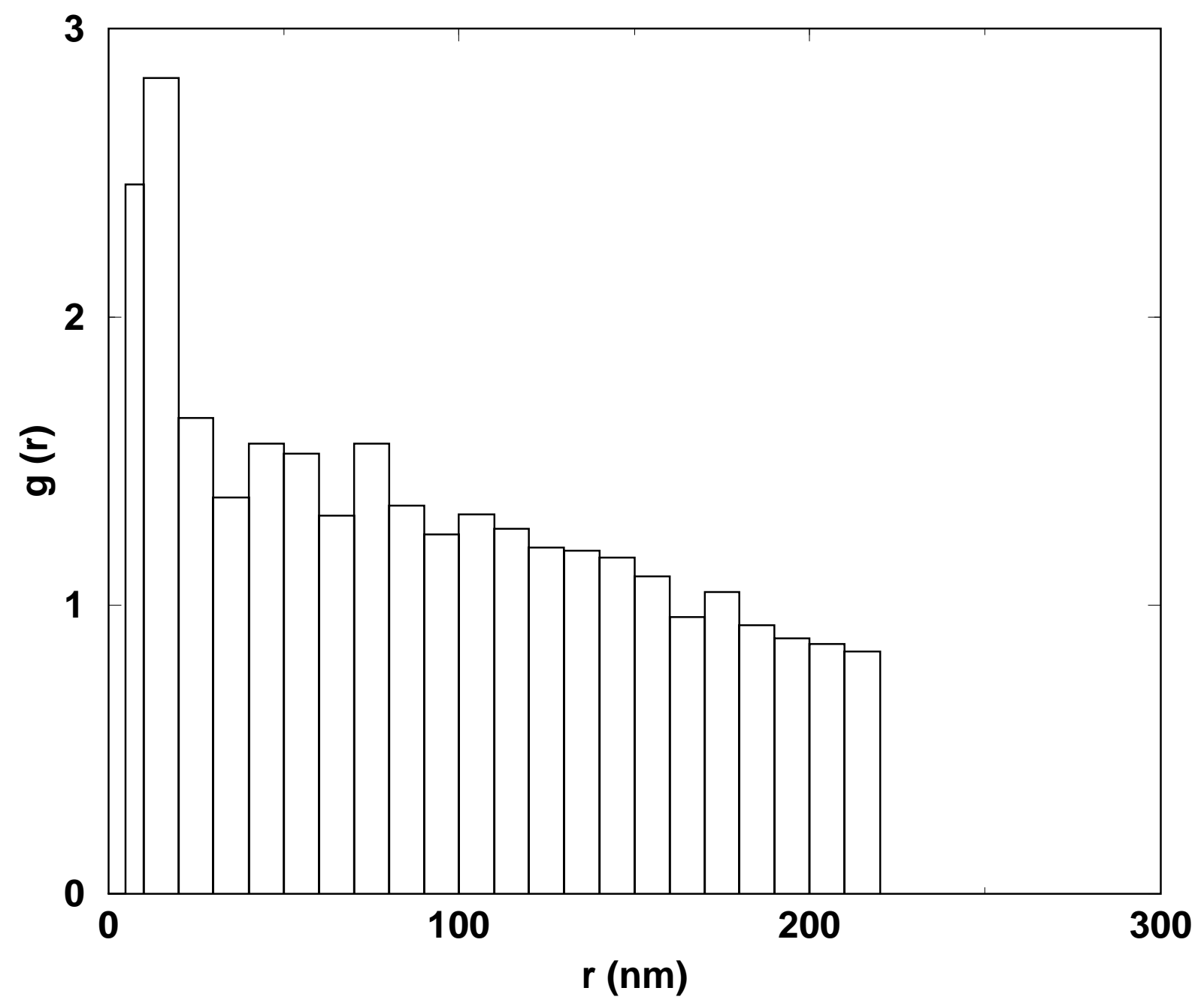

